\title{
Infections associées à des instruments dans les hôpitaux de soins actifs du Canada de 2009 à 2018
}

\author{
Programme canadien de surveillance des infections nosocomiales ${ }^{1}$
}

\section{Resumé}

Contexte : Les infections associées aux soins de santé posent un risque grave pour la sécurité des patients et la qualité des soins. Le Programme canadien de surveillance des infections nosocomiales effectue une surveillance nationale des infections associées aux soins de santé dans les hôpitaux sentinelles de soins actifs partout au Canada. Le présent rapport donne un aperçu de 10 années de données canadiennes sur l'épidémiologie de certaines infections associées aux soins de santé associées à des instruments.

Méthodes : Plus de 40 hôpitaux ont soumis des données entre 2009 et 2018 sur les infections du site opératoire de la hanche et du genou, les infections du site opératoire de dérivation des liquides céphalorachidiens, les infections du site opératoire cardiaques pédiatriques et les infections du système sanguin associées aux cathéters centraux. Les chiffres, les taux, les caractéristiques des patients et de l'hôpital, ainsi que les distributions de pathogènes et les susceptibilités aux antimicrobiens sont présentés.

Résultats : Au total, 4300 infections associées à des instruments ont été signalées. Les infections du système sanguin associées aux cathéters centraux étaient les infections associées aux soins de santé associées aux instruments le plus souvent signalées ( $n=2973,69 \%$ ) et les infections par arthroplastie de la hanche et du genou étaient les infections du site opératoire les plus courantes déclarées ( $66 \%$ des infections du site opératoire). Nos résultats montrent une diminution des taux d'infections du système sanguin associées aux cathéters centraux dans les unités de soins intensifs néonatals (de 4,2 à 1,9 par 1000 jours, $p<0,0001$ ) et une diminution des taux d'infections du site opératoire au genou (de 0,69 à 0,30 infection par 100 opérations, $p=0,007)$. Les taux d'infections associées aux soins de santé associées aux instruments sont demeurés relativement constants au cours de la période de surveillance de 10 ans. Dans l'ensemble, 4599 agents pathogènes ont été décelés à partir d'infections associées aux soins de santé associées à des instruments; $70 \%$ d'entre eux étaient liés à des infections du système sanguin associées aux cathéters centraux. Les staphylocoques à coagulase négative (29\%) et le Staphylococcus aureus (14\%) étaient les pathogènes les plus fréquemment déclarés. Les pathogènes à Gram positif représentaient $68 \%$ des pathogènes décelés, les pathogènes à Gram négatif représentaient $22 \%$ et les pathogènes fongiques représentaient $9 \%$.

Conclusion : Il est essentiel de comprendre le fardeau national des infections associées aux soins de santé associées aux instruments pour élaborer et maintenir des taux de référence afin d'éclairer les politiques et les programmes de contrôle des infections et de prévention des antimicrobiens.
Citation proposée : Programme canadien de surveillance des infections nosocomiales. Infections associées à des instruments dans les hôpitaux de soins actifs du Canada de 2009 à 2018. Relevé des maladies transmissibles au Canada 2020;46(11/12):435-48. https://doi.org/10.14745/ccdr.v46i1112a05f

Mots-clés : infection dans les hôpitaux, soins actifs, surveillance, résistance aux antimicrobiens, infections associées à un instrument, infections du site opératoire, Canada
Cette oeuvre est mise à la disposition selon les termes de la licence internationale Creative Commons Attribution 4.0

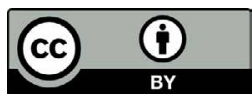

Affiliation

${ }^{1}$ Agence de la santé publique du Canada, Ottawa, ON

*Correspondance :

phac.cnisp-pcsin.aspc@canada.ca 


\section{Introduction}

Les infections associées aux soins de santé posent un risque grave pour la sécurité des patients et la qualité des soins, contribuent à prolonger les séjours à l'hôpital, à accroître la résistance aux antimicrobiens, à augmenter les coûts pour le système de santé et à entraîner des décès inutiles (1). Les facteurs de risque des infections associées aux soins de santé comprennent l'utilisation d'instruments effractifs, les interventions chirurgicales et l'utilisation inappropriée d'antibiotiques (2). Au Canada, les infections du site opératoire touchent environ 26000 à 65000 patients par année (3). Dans une étude canadienne datant de 2017 sur la prévalence ponctuelle dans les hôpitaux sentinelles, les infections associées à des instruments représentaient $35,6 \%$ de toutes les infections associées aux soins de santé signalées. Parmi les infections associées à un instrument, les infections du site opératoire associées à un implant prothétique représentaient 19,4\% des cas et les infections du système sanguin associées aux cathéters centraux représentaient $21,2 \%$ des cas (4).

Les renseignements sur la susceptibilité aux antimicrobiens associée aux infections associées aux soins de santé ont d'importantes répercussions sur la résistance aux antibiotiques (5), ce qui a une incidence sur la durée du séjour et les coûts des soins de santé (6). Les antibiogrammes cumulatifs sont une ressource précieuse pour la prise de décisions cliniques pendant que les résultats de sensibilité sont en attente (7). Le risque d'infections associées aux soins de santé associées à un instrument varie selon les populations de patients et les types d'hôpitaux; les patients admis aux unités de soins intensifs courent un risque plus élevé de contracter une infection associée aux soins de santé (8).

Il est essentiel de comprendre les tendances des infections associées aux soins de santé associées aux instruments pour prévenir et contrôler efficacement les infections. S'appuyant sur une décennie de données sur les infections associées aux soins de santé (2009 à 2018) provenant de plus de 40 hôpitaux sentinelles de soins actifs du Canada qui participent au Programme canadien de surveillance des infections nosocomiales, le présent rapport donne un aperçu épidémiologique de certaines infections associées aux soins de santé associées à des instruments.

\section{Méthodes}

\section{Conception}

Créé en 1994, le Programme canadien de surveillance des infections nosocomiales, une collaboration entre l'Agence de la santé publique du Canada, I'Association pour la microbiologie médicale et l'infectiologie Canada et les hôpitaux sentinelles du Canada, assure la surveillance nationale des infections associées aux soins de santé dans les hôpitaux sentinelles de soins actifs du Canada. Ce rapport présente des données sur les infections associées aux soins de santé associées aux instruments pour les infections suivantes: infections du site opératoire de la hanche et du genou; infections du site opératoire des voies de dérivation du liquide céphalorachidien; infections du site opératoire après une opération cardiaque pédiatrique et infections du système sanguin associées aux cathéters centraux.

\section{Définitions de cas}

Les infections associées aux soins de santé associées aux instruments ont été définies selon des protocoles normalisés et des définitions de cas examinées par des experts (annexe 1). Seules les infections du système sanguin associées aux cathéters centraux décelées dans les unités de soins intensifs ont été incluses dans la surveillance. Seules les infections complexes, définies comme une infection du site opératoire profonde associée à une incision et l'espace des organes, ont été incluses dans la surveillance des infections du site opératoire de la hanche et du genou.

\section{Source des données}

Entre le $1^{\text {er }}$ janvier 2009 et le 31 décembre 2018, les hôpitaux participants ont présenté des données épidémiologiques sur les infections du site opératoire de dérivation du liquide céphalorachidien et les infections du système sanguin associées aux cathéters centraux. La surveillance des infections du site opératoire cardiaques pédiatriques a commencé en janvier 2010. La surveillance des infections du site opératoire de la hanche et du genou a commencé en janvier 2011. La présentation des données et l'identification des cas ont été appuyées par des séances de formation annuelles et des évaluations continues de la qualité des données.

\section{Analyse statistique}

Les taux d'infections du système sanguin associées aux cathéters centraux ont été calculés en divisant le nombre de cas par les dénominateurs jours cathéter veineux central (CVC). Les infections du site opératoire de la hanche et du genou, les infections du site opératoire de dérivation du liquide céphalorachidien et les infections du site opératoire cardiaques pédiatriques ont été calculées en divisant le nombre de cas par les dénominateurs de chirurgie. Les proportions des pathogènes ont été calculées en divisant le nombre de pathogène par le nombre total de pathogènes décelés. Les données manquantes et incomplètes ont été exclues des analyses, de sorte que les dénominateurs peuvent varier. Les écarts interquartiles ont été calculés. Le test Mann-Kendall ou de régression binomiale négative a été utilisé pour vérifier les tendances au fil du temps. Les essais de signification étaient bidimensionnels et les différences étaient considérées comme importantes à la valeur $p \leq 0,05$. Les analyses ont été effectuées à l'aide d'Excel et de SAS 9.4. 


\section{Résultats}

Entre 2009 et 2018, plus de 40 hôpitaux ont fourni des données sur les infections associées aux soins de santé associées à des instruments au Programme canadien de surveillance des infections nosocomiales, dont la plupart étaient des hôpitaux de taille moyenne pour adultes (201 à 499 lits) (tableau 1). Dans l'ensemble, 4300 infections associées à des instruments ont été signalées. Les infections du système sanguin associées aux cathéters centraux étaient les infections associées aux soins de santé associées aux instruments les plus courantes ( $n=2973,69 \%$ ). Les infections du site opératoire de la hanche et du genou étaient le type le plus courant d'infections du site opératoire signalées ( $66 \%$ des infections du site opératoire, $n=871 / 1327$ ).

Tableau 1 : Caractéristiques des hôpitaux de soins actifs participant à la surveillance des infections associées aux soins de santé associées aux instruments et fréquence des infections nosocomiales associées aux instruments, 2009 à 2018

\begin{tabular}{|c|c|c|c|c|c|c|c|}
\hline $\begin{array}{l}\text { Caractéristiques } \\
\text { des hôpitaux }\end{array}$ & $\begin{array}{l}\text { ISO de } \\
\text { dérivation } \\
\text { du LCR }\end{array}$ & $\begin{array}{c}\text { ISO } \\
\text { cardiaque } \\
\text { pédiatrique }\end{array}$ & $\begin{array}{c}\text { ISO } \\
\text { de la } \\
\text { hanche } \\
\text { et du } \\
\text { genou }\end{array}$ & $\begin{array}{l}\text { ISSACC } \\
\text { - USI } \\
\text { mixtes } \\
\text { pour } \\
\text { adultes }\end{array}$ & $\begin{array}{l}\text { ISSACC } \\
\text { - USICC } \\
\text { pour } \\
\text { adultes }\end{array}$ & $\begin{array}{l}\text { ISSACC } \\
\text { - USIP }\end{array}$ & $\begin{array}{l}\text { ISSACC - } \\
\text { USIN }\end{array}$ \\
\hline $\begin{array}{l}\text { Années de } \\
\text { surveillance }\end{array}$ & $\begin{array}{c}2009 \text { à } \\
2018\end{array}$ & $\begin{array}{c}2010 \text { à } \\
2018\end{array}$ & $\begin{array}{c}2011 \text { à } \\
2018\end{array}$ & $\begin{array}{c}2009 \text { à } \\
2018\end{array}$ & $\begin{array}{c}2009 \text { à } \\
2018\end{array}$ & $\begin{array}{c}2009 \text { à } \\
2018\end{array}$ & $\begin{array}{c}2009 \text { à } \\
2018\end{array}$ \\
\hline $\begin{array}{l}\text { Nombre d'IASS } \\
\text { signalées }\end{array}$ & 266 & 190 & 871 & 1331 & 192 & 348 & 1102 \\
\hline $\begin{array}{l}\text { Total des } \\
\text { hôpitaux } \\
\text { participants }\end{array}$ & 8 à 14 & 3 à 4 & 12 à 25 & 22 à 41 & 5 à 8 & 5 à 10 & 9 à 17 \\
\hline \multicolumn{8}{|l|}{ Type d'hôpital } \\
\hline Adultes $^{\mathrm{a}}$ & 2 à 5 & s.o. & 8 à 16 & 12 à 27 & 3 à 7 & s.o. & 2 à 3 \\
\hline Mixte & 2 à 4 & s.o. & 4 à 9 & 4 à 14 & 1 à 2 & 0 à 4 & 1 à 6 \\
\hline Pédiatrique & 4 à 7 & 3 à 4 & s.o. & s.o. & s.o. & 4 à 6 & 4 à 8 \\
\hline \multicolumn{8}{|l|}{ Taille de l'hôpital } \\
\hline $\begin{array}{l}\text { Petite } \\
\text { (1 à } 200 \text { lits) }\end{array}$ & 3 à 7 & 2 à 4 & 1 à 2 & 1 à 4 & 0 à 1 & 3 à 5 & 4 à 7 \\
\hline $\begin{array}{l}\text { Moyenne } \\
\text { (201 à } 499 \text { lits) }\end{array}$ & 4 à 8 & 1 & 7 à 15 & 10 à 27 & 2 à 4 & 1 à 5 & 1 à 7 \\
\hline $\begin{array}{l}\text { Grande } \\
\text { (Plus de } 500 \\
\text { lits) }\end{array}$ & 0 à 1 & s.o. & 5 à 8 & 5 à 10 & 2 à 3 & 0 & 1 à 3 \\
\hline $\begin{array}{l}\text { Nombre total } \\
\text { de lits (2018) }\end{array}$ & 3558 & 693 & 9973 & $\begin{array}{r}16701 \\
\text { lits aux } \\
\text { USI }\end{array}$ & $\begin{array}{r}3570 \\
\text { lits aux } \\
\text { USI }\end{array}$ & $\begin{array}{r}2209 \\
\text { lits aux } \\
\text { USI }\end{array}$ & $\begin{array}{r}5500 \text { lits } \\
\text { aux USI }\end{array}$ \\
\hline
\end{tabular}

Abréviations : IASS, infections associées aux soins de santé; ISO, infection du site opératoire; ISO de dérivation du LCR, ISO des voies de dérivation du liquide céphalorachidien; ISSACC, infections du système sanguin associées aux cathéters centraux; s.o., sans objet; USI, unité de soins intensifs; USICC, unité de soins intensifs en chirurgie cardiovasculaire; USIN, unité de soins intensifs néonatales; USIP, unité de soins intensifs pédiatriques

a Sept hôpitaux classés pour "Adultes » avaient également une USIN

Dans l'ensemble, 4599 pathogènes ont été repérés dans des cas d'infections associées aux soins de santé associées à des instruments entre 2014 et $2018 ; 69,8 \%$ de ces cas étaient liés à des infections du système sanguin associées aux cathéters centraux. Les staphylocoques à coagulase négative et le Staphylococcus aureus étaient les pathogènes les plus fréquemment déclarés (tableau 2). Les pathogènes à Gram positif représentaient $68,3 \%$ des pathogènes décelés, les pathogènes à Gram négatif représentaient $22,3 \%$ et les pathogènes fongiques représentaient $9,4 \%$.

Tableau 2 : Répartition et rang des cinq pathogènes à Gram négatif, à Gram positif et des fongiques les plus fréquemment signalés 2009 à $2018^{b}$

\begin{tabular}{|c|c|c|c|c|}
\hline Catégorie & Rang & Agent pathogène & $\mathbf{N}$ & $\begin{array}{c}\% \text { du } \\
\text { total des } \\
\text { pathogènes } \\
\text { décelés }\end{array}$ \\
\hline \multirow[t]{6}{*}{ Gram positif } & 1 & $\begin{array}{l}\text { Staphylocoques à } \\
\text { coagulase négative }^{c}\end{array}$ & 1320 & 28,7 \\
\hline & 2 & Staphylococcus aureus ${ }^{d}$ & 653 & 14,2 \\
\hline & 3 & Enterococcus spp. & 519 & 11,3 \\
\hline & 4 & Streptocoque & 137 & 3,0 \\
\hline & 5 & $\begin{array}{l}\text { S. aureus résistant à la } \\
\text { méthicilline }\end{array}$ & 120 & 2,6 \\
\hline & \multicolumn{2}{|c|}{ Autre à Gram positif } & 392 & 8,5 \\
\hline \multirow[t]{6}{*}{ Gram négatif } & 1 & Klebsiella spp. & 226 & 4,9 \\
\hline & 2 & Escherichia coli & 197 & 4,3 \\
\hline & 3 & Enterobacter & 170 & 3,7 \\
\hline & 4 & Pseudomonas aeruginosa & 133 & 2,9 \\
\hline & 5 & Serratia & 87 & 1,9 \\
\hline & \multicolumn{2}{|c|}{ Autre à Gram négatif } & 214 & 4,7 \\
\hline \multirow[t]{3}{*}{ Fongiques } & 1 & Candida albicans & 210 & 4,6 \\
\hline & 2 & Autres Candida spp. & 199 & 4,3 \\
\hline & \multicolumn{2}{|c|}{ Autres fongiques } & 22 & 0,5 \\
\hline \multicolumn{3}{|l|}{ Total } & 4599 & $100,0^{\circ}$ \\
\hline
\end{tabular}

Jusqu'à quatre pathogènes par infection nosocomiale associée à un instrument ont été inclus dans l'analyse

b La surveillance des infections du site opératoire cardiaques pédiatriques a commencé en 2010. La surveillance des infections du site opératoire de la hanche et du genou a commencé en 2011 ' Les staphylocoques à coagulase négative comprennent S. lugdunesis, S. haemolyticus, S. epidermidis et $S$. capitis

Le Staphylococcus aureus comprend le $S$. aureus sensible à la méthicilline et le $S$. aureus non spécifié

e Pourcentage arrondi au nombre entier le plus près

\section{Infections du système sanguin associées aux cathéters centraux}

Entre 2009 et 2018, il y a eu 2973 infections du système sanguin associées aux cathéters centraux déclarées, dont la majorité se sont produites dans des unités de soins intensifs mixtes pour adultes ( $n=1331,44,8 \%$ ) et des unités de soins intensifs néonatales ( $n=1102,37,1 \%$ ). Parmi les infections du système sanguin associées aux cathéters centraux recensées dans les unités de soins intensifs pour adultes, l'âge médian était de 63 ans (écarts interquartiles = 52 à 73 ans). Les hommes représentaient $62 \%$ des infections du système sanguin associées aux cathéters centraux pour les adultes. Un tiers des patients adultes atteints d'infections du système sanguin associées aux cathéters centraux sont décédés dans les 30 jours suivant la 
première culture positive $(32,3 \%, n=482 / 1492)$. Parmi les infections du système sanguin associées aux cathéters centraux recensées dans les unités de soins intensifs pédiatriques, l'âge médian était de six mois (écarts interquartiles $=2$ à 22 mois). Les patients de sexe masculin représentaient $51 \%$ des cas d'unités de soins intensifs pédiatriques et dans les 30 jours suivant une culture positive, $11 \%$ des patients infectés étaient décédés ( $n=37 / 342$ ). Parmi les infections du système sanguin associées aux cathéters centraux décelées dans l'unité de soins intensifs néonatales, l'âge médian à la première culture positive était de 20 jours (écarts interquartiles $=10$ à 45 jours). Les patients de sexe masculin représentaient $57 \%$ des cas d'unité de soins intensifs néonatales et dans les 30 jours suivant la culture positive, $8 \%$ des patients infectés étaient décédés $(n=88 / 1$ 077).

Dans l'ensemble, les unités de soins intensifs néonatales affichaient des taux plus élevés d'infections du système sanguin associées aux cathéters centraux (2,7 cas par 1000 jours CVC, en moyenne) que les unités de soins intensifs pédiatriques (1,9 par 1000 jours CVC), les unités de soins intensifs mixtes adultes (1,1 par 1000 jours CVC) et les unités de soins intensifs en chirurgie cardiovasculaire pour adultes $(0,7$ par 1000 jours CVC). Bien que les taux soient demeurés relativement constants pour les unités de soins intensifs adultes et les unités de soins intensifs pédiatriques, une diminution de $54,8 \%$ a été observée parmi les unités de soins intensifs néonatales (de 4,2 à 1,9 par 1000 jours CVC, de 2009 à 2018, p < 0,0001) (tableau 3).

Tableau 3 : Taux d'infections du système sanguin associées aux cathéters centraux par 1000 jours CVC, par type d'unité de soins intensifs, 2009 à 2018

\begin{tabular}{|c|c|c|c|c|}
\hline \multirow[b]{2}{*}{ Année } & \multicolumn{4}{|c|}{ Taux d'ISSACC par 1000 jours CVC } \\
\hline & $\begin{array}{c}\text { USI mixtes } \\
\text { pour } \\
\text { adultes }\end{array}$ & $\begin{array}{l}\text { USICC } \\
\text { pour } \\
\text { adultes }\end{array}$ & USIN & USIP \\
\hline 2009 & 1,4 & 0,8 & 4,2 & 2,0 \\
\hline 2010 & 1,1 & 0,9 & 3,9 & 1,7 \\
\hline 2011 & 0,9 & 1,0 & 4,1 & 1,6 \\
\hline 2012 & 1,0 & 1,3 & 3,5 & 1,4 \\
\hline 2013 & 1,1 & 0,5 & 2,8 & 1,3 \\
\hline 2014 & 0,9 & 0,5 & 2,1 & 2,0 \\
\hline 2015 & 1,1 & 0,7 & 2,3 & 2,4 \\
\hline 2016 & 1,0 & 0,5 & 2,3 & 1,7 \\
\hline 2017 & 1,2 & 0,4 & 1,8 & 2,0 \\
\hline 2018 & 1,2 & 0,9 & 1,9 & 2,1 \\
\hline $\begin{array}{l}\text { Aperçu } \\
\text { global }\end{array}$ & 1,1 & 0,7 & 2,7 & 1,9 \\
\hline
\end{tabular}

\section{Infections du site opératoire de la hanche et du genou}

Entre 2011 et 2018, 871 infections du site opératoire complexes de la hanche et du genou ont été signalées, dont la majorité étaient des chirurgies de la hanche ( $n=530,60,8 \%)$. On indique que $52 \%(n=455)$ étaient des infections de l'espace des organes et $47,8 \%(n=416)$ étaient des infections profondes associées à une incision (tableau 4). L'âge médian des patients était de 69 et 67 ans pour les infections du site opératoire de la hanche et du genou, respectivement. Le temps médian entre la procédure et l'infection était de 20 jours pour les infections de la hanche et de 22 jours pour les infections du genou. Après la collecte de données supplémentaires à compter de 2018, la durée médiane du séjour pour les chirurgies de la hanche et du genou était de quatre et trois jours, respectivement. On indique que $91 \%$ des patients atteints d'une infection du site opératoire ont subi une arthroplastie de la hanche ou du genou (hanche : $n=83 / 91,91,2 \%$; genou : $n=33 / 37,89,1 \%$ ) et $64,8 \%$ ( $n=83 / 128)$ ont dû subir une intervention chirurgicale de révision. À 30 jours après une chirurgie, un décès a été signalé en 2018 chez les patients atteints d'une infection du site opératoire de la hanche.

\section{Tableau 4 : Fréquence des infections du site opératoire de la hanche et du genou par type et taux par 100 interventions chirurgicales, 2011 à 2018}

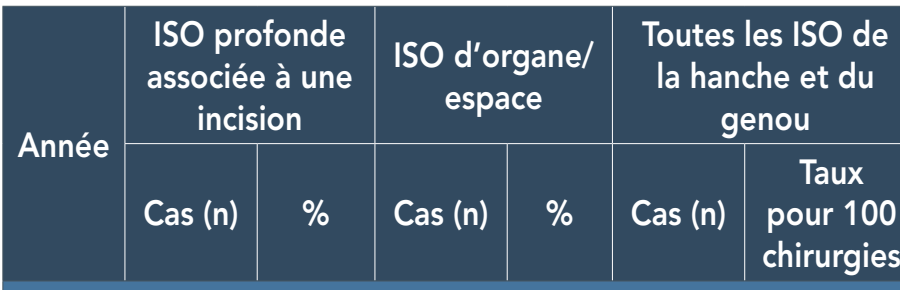

Arthroplastie de la hanche

\begin{tabular}{|l|r|r|r|r|r|r|}
\hline 2011 & 18 & 43,9 & 23 & 56,1 & 41 & 0,82 \\
\hline 2012 & 32 & 66,7 & 16 & 33,3 & 48 & 0,73 \\
\hline 2013 & 36 & 57,1 & 27 & 42,9 & 63 & 0,79 \\
\hline 2014 & 36 & 50,7 & 35 & 49,3 & 71 & 0,85 \\
\hline 2015 & 34 & 51,5 & 32 & 48,5 & 66 & 0,75 \\
\hline 2016 & 28 & 41,2 & 40 & 58,8 & 68 & 0,79 \\
\hline 2017 & 34 & 41,5 & 48 & 58,5 & 82 & 0,80 \\
\hline 2018 & 29 & 31,9 & 62 & 68,1 & 91 & 0,87 \\
\hline $\begin{array}{l}\text { Aperçu } \\
\text { général }\end{array}$ & 247 & 46,6 & 283 & 53,4 & 530 & 0,80 \\
\hline
\end{tabular}

Arthroplastie du genou

\begin{tabular}{|l|l|l|l|l|l|l|}
\hline 2011 & 20 & 51,3 & 19 & 48,7 & 39 & 0,69 \\
\hline 2012 & 26 & 52,0 & 24 & 48,0 & 50 & 0,65 \\
\hline 2013 & 21 & 55,3 & 17 & 44,7 & 38 & 0,41 \\
\hline 2014 & 26 & 48,1 & 28 & 51,9 & 54 & 0,56 \\
\hline
\end{tabular}


Tableau 4 : Fréquence des infections du site opératoire de la hanche et du genou par type et taux par 100 interventions chirurgicales, 2011 à 2018 (suite)

\begin{tabular}{|l|r|r|r|r|r|r|}
\hline \multirow{2}{*}{ Année } & $\begin{array}{c}\text { ISO profonde } \\
\text { associée à une } \\
\text { incision }\end{array}$ & \multicolumn{2}{|c|}{$\begin{array}{c}\text { ISO d'organe/ } \\
\text { espace }\end{array}$} & $\begin{array}{r}\text { Toutes les ISO de } \\
\text { la hanche et du } \\
\text { genou }\end{array}$ \\
\cline { 2 - 7 } & Cas (n) & $\%$ & Cas (n) & $\%$ & Cas (n) & \multicolumn{2}{|c|}{$\begin{array}{c}\text { Taux } \\
\text { pour 100 } \\
\text { chirurgies }\end{array}$} \\
\hline Arthroplastie du genou (suite) & & & & \\
\hline 2015 & 21 & 47,7 & 23 & 52,3 & 44 & 0,43 \\
\hline 2016 & 15 & 41,7 & 21 & 58,3 & 36 & 0,35 \\
\hline 2017 & 20 & 46,5 & 23 & 53,5 & 43 & 0,36 \\
\hline 2018 & 20 & 54,1 & 17 & 45,9 & 37 & 0,30 \\
\hline $\begin{array}{l}\text { Aperçu } \\
\text { global }\end{array}$ & 169 & 49,6 & 172 & 50,4 & 341 & 0,47 \\
\hline
\end{tabular}

De 2011 à 2018, le taux d'infection du site opératoire de la hanche était stable (de 0,82 à 0,87 infection par 100 interventions chirurgicales, $p=0,26$ ), tandis que le taux d'infection du site opératoire du genou a considérablement diminué (de 0,69 à 0,30 infection par 100 interventions chirurgicales, $p=0,007)$. $S$. aureus et les staphylocoques négatifs à la coagulase étaient les pathogènes les plus fréquemment décelés dans les cas d'infection du site opératoire de la hanche et du genou (32\% et $17 \%$ des pathogènes décelés, respectivement).

\section{Infections du site opératoire des voies de dérivation du liquide céphalorachidien}

Entre 2009 et 2018, 266 infections du site opératoire de dérivation du liquide céphalorachidien ont été signalées, 143 sur $260(55 \%)$ ont été décelées à partir de nouvelles chirurgies et 117 sur $260(45 \%)$ à partir de chirurgies de révision. L'âge médian des cas était de 46 ans (écarts interquartiles $=29$ à 67 ans) pour les patients adultes et de 0,6 an (écarts interquartiles $=0,2$ à 6,8 ans) pour les patients pédiatriques. Les patientes représentaient $53,4 \%(n=140 / 262)$ des cas. Le nombre médian de jours entre la chirurgie et l'infection était de 29 jours (écarts interquartiles $=14$ à 64 jours).

De 2009 à 2018, le taux global d'infections du site opératoire de dérivation du liquide céphalorachidien était de 3,2 par 100 interventions chirurgicales (fourchette : 1,9 à 5,7 par 100 chirurgies, tableau 5). Les taux d'infection étaient semblables dans les hôpitaux pédiatriques ( $n=3,3$ par 100 chirurgies) et les hôpitaux pour adultes/mixtes ( $n=3,2$ par 100 chirurgies). Les staphylocoques négatifs à la coagulase et $S$. aureus étaient les pathogènes les plus fréquemment décelés dans les infections du site opératoire de dérivation du liquide céphalorachidien ( $41 \%$ et $22 \%$ des pathogènes décelés, respectivement).
Tableau 5 : Taux d'infections du site opératoire des voies de dérivation du liquide céphalorachidien par 100 interventions chirurgicales, par type d'hôpital, 2009 à 2018

\begin{tabular}{|l|r|r|r|}
\hline \multirow{2}{*}{ Année } & \multicolumn{3}{|c|}{ Taux par 100 chirurgies } \\
\cline { 2 - 4 } & $\begin{array}{c}\text { Hôpitaux pour } \\
\text { adultes et } \\
\text { hôpitaux mixtes }\end{array}$ & $\begin{array}{c}\text { Hôpitaux } \\
\text { pédiatriques }\end{array}$ & $\begin{array}{c}\text { Tous les } \\
\text { hôpitaux }\end{array}$ \\
\hline 2009 & 2,9 & 2,8 & 2,9 \\
\hline 2010 & 3,2 & 3,9 & 3,5 \\
\hline 2011 & 5,0 & 6,3 & 5,7 \\
\hline 2012 & 2,5 & 3,9 & 3,2 \\
\hline 2013 & 2,6 & 2,8 & 2,7 \\
\hline 2014 & 1,6 & 2,6 & 2,0 \\
\hline 2015 & 3,3 & 2,1 & 2,7 \\
\hline 2016 & 4,4 & 2,4 & 3,3 \\
\hline 2017 & 4,6 & 3,2 & 3,9 \\
\hline 2018 & 2,4 & 2,3 & 2,4 \\
\hline Aperçu & 3,2 & 3,3 & 3,2 \\
global & & & \\
\hline
\end{tabular}

\section{Infections des sites opératoires après une opération cardiaque pédiatrique}

Entre 2010 et 2018, 190 infections du site opératoire pédiatriques cardiaques ont été signalées (tableau 6). La plupart des cas étaient des infections superficielles $(58,7 \%)$ ou des infections d'organes ou d'espace (32,3\%). L'âge moyen des patients atteints d'une infection du site opératoire pédiatrique cardiaque était de 19 jours (écarts interquartiles $=7$ à 213 jours). En moyenne, le temps écoulé entre la chirurgie et la date d'apparition de l'infection était de 10 jours (écarts interquartiles $=5$ à 19 jours). On a signalé trois décès dans les 30 jours suivant l'apparition de l'infection (1,6\% des cas), mais les trois décès n'étaient pas liés à l'infections du site opératoire pédiatrique cardiaque.

Dans l'ensemble, le taux d'infections du site opératoire pédiatrique cardiaque moyen était de 4,1 par 100 chirurgies. Bien que les taux soient demeurés généralement constants $(p=0,35)$, il y a eu une augmentation importante en $2018(n=7,5$ par 100 chirurgies, $p<0,001)$ comparativement aux taux globaux de 2010 à 2017 (3,6 par 100 chirurgies). $S$. aureus et les staphylocoques négatifs à la coagulase étaient les pathogènes les plus fréquemment décelés pour les infections du site opératoire pédiatriques cardiaques ( $43 \%$ et $24 \%$ des pathogènes décelés, respectivement).

\section{Antibiogramme}

Les résultats des tests de sensibilité aux antimicrobiens pour les pathogènes à Gram positif, à Gram négatif et fongiques les plus infections associées aux soins de santé fréquemment décelés dans les associées aux instruments sont énumérés dans le tableau 7. La résistance à l'oxacilline et à la cycloxacilline a été 
Tableau 6 : Taux d'infection du site opératoire après une opération cardiaque pédiatrique selon l'année et le type d'infection, 2010 à 2018

\begin{tabular}{|c|c|c|c|c|c|c|c|c|}
\hline \multirow[t]{2}{*}{ Année } & \multicolumn{2}{|c|}{ Superficiel } & \multicolumn{2}{|c|}{ Organe/espace } & \multicolumn{2}{|c|}{ Profond } & \multicolumn{2}{|c|}{$\begin{array}{l}\text { Toutes les infections du site } \\
\text { opératoire après une opération } \\
\text { cardiaque pédiatrique }\end{array}$} \\
\hline & Nombre de cas & $\%$ de cas annuels & Nombre de cas & $\%$ de cas annuels & Nombre de cas & $\%$ de cas annuels & $\begin{array}{c}\text { Nombre de } \\
\text { cas }\end{array}$ & $\begin{array}{c}\text { Taux par } 100 \\
\text { chirurgies }\end{array}$ \\
\hline 2010 & 9 & 40,9 & 10 & 45,5 & 3 & 13,6 & 22 & 4,1 \\
\hline 2011 & 8 & 53,3 & 5 & 33,3 & 2 & 13,3 & 15 & 3,1 \\
\hline 2012 & 15 & 83,3 & 2 & 11,1 & 1 & 5,6 & 18 & 2,9 \\
\hline $2013^{a}$ & 12 & 63,2 & 7 & 36,8 & 0 & 0,0 & 19 & 4,6 \\
\hline 2014 & 11 & 57,9 & 8 & 42,1 & 0 & 0,0 & 19 & 3,5 \\
\hline 2015 & 12 & 63,2 & 6 & 31,6 & 1 & 5,3 & 19 & 3,5 \\
\hline 2016 & 9 & 64,3 & 3 & 21,4 & 2 & 14,3 & 14 & 3,0 \\
\hline 2017 & 17 & 70,8 & 5 & 20,8 & 2 & 8,3 & 24 & 4,4 \\
\hline 2018 & 18 & 46,2 & 15 & 38,5 & 6 & 15,4 & 40 & 7,5 \\
\hline $\begin{array}{l}\text { Aperçu } \\
\text { global }\end{array}$ & 111 & 58,7 & 61 & 32,3 & 17 & 9,0 & 190 & 4,1 \\
\hline
\end{tabular}

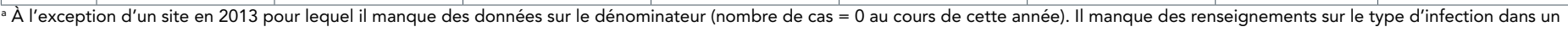
cas

Tableau 7 : Résultats de l'antibiogramme aux agents pathogènes décelés à partir d'infections associées aux soins de santé associées à des instruments, 2014 à 2018

\begin{tabular}{|c|c|c|c|c|c|c|c|c|c|c|c|c|c|c|c|c|}
\hline \multirow{4}{*}{ Antibiotique } & \multicolumn{16}{|c|}{ Nombre de résistants/nombre de tests et $\%$} \\
\hline & \multicolumn{6}{|c|}{ Gram positif } & \multicolumn{6}{|c|}{ Gram négatif } & \multicolumn{4}{|c|}{ Fongiques } \\
\hline & \multicolumn{2}{|c|}{$\begin{array}{c}\text { Staphylocoque } \\
\text { à coagulase } \\
\text { négative }^{b}\end{array}$} & \multicolumn{2}{|c|}{ S. aureus ${ }^{c}$} & \multicolumn{2}{|c|}{$\begin{array}{c}\text { Enterococcus } \\
\text { spp. }\end{array}$} & \multicolumn{2}{|c|}{ Klebsiella spp. } & \multicolumn{2}{|l|}{ E. coli } & \multicolumn{2}{|c|}{ Enterobacter } & \multicolumn{2}{|c|}{ C. albicans } & \multicolumn{2}{|c|}{$\begin{array}{c}\text { Candida spp. } \\
\text { autre }\end{array}$} \\
\hline & $\begin{array}{l}\text { Nombre } \\
\text { de } \\
\text { résistants }\end{array}$ & $\%$ & $\begin{array}{l}\text { Nombre } \\
\text { de } \\
\text { résistants }\end{array}$ & $\%$ & $\begin{array}{l}\text { Nombre } \\
\text { de } \\
\text { résistants }\end{array}$ & $\%$ & $\begin{array}{l}\text { Nombre } \\
\text { de } \\
\text { résistants }\end{array}$ & $\%$ & $\begin{array}{l}\text { Nombre } \\
\text { de } \\
\text { résistants }\end{array}$ & $\%$ & $\begin{array}{l}\text { Nombre } \\
\text { de } \\
\text { résistants }\end{array}$ & $\%$ & $\begin{array}{l}\text { Nombre } \\
\text { de } \\
\text { résistants }\end{array}$ & $\%$ & $\begin{array}{l}\text { Nombre } \\
\text { de } \\
\text { résistants }\end{array}$ & $\%$ \\
\hline Ampicilline & $5 / 8$ & 63 & $2 / 10$ & 20 & $61 / 235$ & 26 & $76 / 78$ & 97 & $56 / 86$ & 65 & $51 / 55$ & 93 & s.o. & s.o. & s.o. & s.o. \\
\hline Céfazoline & $125 / 154$ & 81 & $17 / 158$ & 11 & s.o. & s.o. & $21 / 58$ & 36 & $24 / 76$ & 32 & $47 / 48$ & 98 & s.o. & s.o. & s.o. & s.o. \\
\hline Ceftriaxone & s.o. & s.o. & $1 / 11$ & 9 & s.o. & s.o. & $5 / 62$ & 8 & $11 / 54$ & 20 & $24 / 50$ & 48 & s.o. & s.o. & s.o. & s.o. \\
\hline Clindamycine & $109 / 193$ & 56 & $47 / 213$ & 22 & s.o. & s.o. & s.o. & s.o. & s.o. & s.o. & s.o. & s.o. & s.o. & s.o. & s.o. & s.o. \\
\hline Ciprofloxacine & $1 / 7$ & 14 & $2 / 14$ & 14 & s.o. & s.o. & $6 / 72$ & 8 & $23 / 68$ & 34 & $0 / 64$ & 0 & s.o. & s.o. & s.o. & s.o. \\
\hline Cloxacilline/ & $241 / 308$ & 78 & $38 / 238$ & 16 & s.o. & s.o. & s.o. & s.o. & s.o. & s.o. & s.o. & s.o. & s.o. & s.o. & s.o. & s.o. \\
\hline Oxacilline & $57 / 89$ & 64 & $34 / 104$ & 33 & s.o. & s.o. & s.o. & s.o. & s.o. & s.o. & s.o. & s.o. & s.o. & s.o. & s.o. & s.o. \\
\hline Érythromycine & $15 / 31$ & 48 & $2 / 27$ & 7 & $7 / 58$ & 12 & $6 / 84$ & 7 & $11 / 81$ & 14 & $1 / 14$ & 7 & s.o. & s.o. & s.o. & s.o. \\
\hline Gentamicine & s.o. & s.o. & s.o. & s.o. & s.o. & s.o. & $2 / 36$ & 6 & $1 / 33$ & 3 & $0 / 36$ & 0 & s.o. & s.o. & s.o. & s.o. \\
\hline Méropénème & s.o. & s.o. & s.o. & s.o. & s.o. & s.o. & $7 / 60$ & 12 & $12 / 60$ & 20 & $21 / 48$ & 44 & s.o. & s.o. & s.o. & s.o. \\
\hline $\begin{array}{l}\text { Pipéracilline- } \\
\text { tazobactame }\end{array}$ & $85 / 87$ & 98 & $81 / 86$ & 94 & s.o. & s.o. & s.o. & s.o. & s.o. & s.o. & s.o. & s.o. & s.o. & s.o. & s.o. & s.o. \\
\hline Pénicilline & $1 / 59$ & 2 & $0 / 33$ & 0 & s.o. & s.o. & s.o. & s.o. & s.o. & s.o. & s.o. & s.o. & s.o. & s.o. & s.o. & s.o. \\
\hline Rifampicine & $58 / 147$ & 39 & $4 / 177$ & 2 & s.o. & s.o. & $5 / 56$ & 9 & $28 / 59$ & 47 & $9 / 53$ & 17 & s.o. & s.o. & s.o. & s.o. \\
\hline $\begin{array}{l}\text { Triméthoprime- } \\
\text { sulfaméthoxazole }\end{array}$ & s.o. & s.o. & s.o. & s.o. & s.o. & s.o. & $6 / 72$ & 8 & $3 / 80$ & 4 & $2 / 61$ & 3 & s.o. & s.o. & s.o. & s.o. \\
\hline Tobramycine & $3 / 293$ & 1 & $1 / 140$ & 1 & $32 / 187$ & 17 & s.o. & s.o. & s.o. & s.o. & s.o. & s.o. & s.o. & s.o. & s.o. & s.o. \\
\hline Vancomycine & s.o. & s.o. & s.o. & s.o. & s.o. & s.o. & s.o. & s.o. & s.o. & s.o. & s.o. & s.o. & $0 / 11$ & 0 & $0 / 9$ & 0 \\
\hline Amphotéricine B & s.o. & s.o. & s.o. & s.o. & s.o. & s.o. & s.o. & s.o. & s.o. & s.o. & s.o. & s.o. & $1 / 40$ & 3 & $0 / 11$ & 0 \\
\hline Caspofungine & s.o. & s.o. & s.o. & s.o. & s.o. & s.o. & s.o. & s.o. & s.o. & s.o. & s.o. & s.o. & $1 / 55$ & 2 & $19 / 59$ & 32 \\
\hline Fluconazole & s.o. & s.o. & s.o. & s.o. & s.o. & s.o. & s.o. & s.o. & s.o. & s.o. & s.o. & s.o. & $1 / 55$ & 2 & $19 / 59$ & 32 \\
\hline
\end{tabular}

Abréviation : s.o., sans objet

a Les combinaisons antibiotique/organisme comportant moins de six tests ont été exclues

b Les staphylocoques négatifs à la coagulase comprennent S. lugdunesis, S. haemolyticus, S. epidermidis, S. capitis et S. warner

Comprend le $S$. aureus sensible à la méthicilline et le $S$. aureus résistant à la méthicilline 
constatée dans $13 \%(n=38 / 288)$ de tous les isolats de $S$. aureus. La résistance au méropénème était faible chez les pathogènes à Gram négatif, avec deux isolats de Klebsiella sur 36, un isolat $\mathrm{d}^{\prime} E$. coli sur 33 et aucun isolat d'Enterobacter sur 33 résistants au méropénème. On a décelé 32 entérocoques résistants à la vancomycine ( $n=32 / 187,17 \%$, Enterococcus spp.).

\section{Discussion}

Ce rapport décrit les 4300 infections associées aux soins de santé associées à des instruments signalés sur une période de surveillance de 10 ans. À l'exception de la diminution des taux $d$ 'infections du système sanguin associées aux cathéters centraux dans les unités de soins intensifs néonatales et de la diminution des taux d'infections du site opératoire du genou, les taux d'infections associées aux soins de santé associées aux instruments sont demeurés relativement constants. En général, les agents pathogènes les plus fréquemment signalés parmi les infections associées aux soins de santé associées à des instruments au Canada s'harmonisent aux résultats obtenus aux États-Unis : S. aureus, E. coli et Klebsiella se sont classés parmi les cinq premiers agents pathogènes dans notre surveillance ainsi que dans un rapport du National Healthcare Surveillance Network des États-Unis de 2020 sur les infections associées aux soins de santé pour adultes (notamment les infections du système sanguin associées aux cathéters centraux, diverses infections du site opératoire, les infections des voies urinaires liées aux cathéters et les événements liés aux ventilateurs) (5).

\section{Infections des sites opératoires}

Les infections du site opératoire de la hanche et du genou étaient les infections du site opératoire les plus fréquemment signalées dans notre surveillance. À l'instar des résultats du Centre européen de prévention et de contrôle des maladies, une tendance à la baisse des infections du site opératoire du genou a été observée dans les hôpitaux du Programme canadien de surveillance des infections nosocomiales, tandis que les infections du site opératoire de la hanche sont demeurées stables (9). De plus, une étude de prévalence ponctuelle aux États-Unis a observé une réduction significative de la prévalence des infections du site opératoire complexes entre 2011 et 2015 (10). Nos constatations indiquent que les agents pathogènes les plus couramment décelés parmi les infections du site opératoire de la hanche et du genou étaient le $S$. aureus et les staphylocoques négatifs à la coagulase, ce qui correspond aux résultats d'autres régions $(9,11)$. L'identification fréquente de $S$. aureus et de staphylocoques négatifs à la coagulase peut être liée à l'utilisation d'implants et à la contamination par la flore cutanée endogène du patient (5). Les infections du site opératoire de la hanche et du genou touchent une population plus âgée, car les arthroplasties surviennent habituellement chez les adultes plus âgés (12). À mesure que les populations vieillissent, les arthroplasties de la hanche et du genou augmentent et sont liées à une augmentation des complications chirurgicales (i.e. infections des articulations prothétiques) (12). Les taux élevés observés de réadmission et de chirurgie de révision mettent en évidence le fardeau financier imposé au système de santé en raison des infections du site opératoire de la hanche et du genou (13).

Notre taux global d'infections du site opératoire de dérivation du liquide céphalorachidien ( $n=3,2$ par 100 chirurgies) se situe à l'extrémité inférieure de ce qui est déclaré à l'échelle internationale; une étude de 2012 a révélé que les taux d'infection déclarés varient de $3 \%$ à $12 \%$ des opérations de dérivation (14). La stratification de nos données sur les infections du site opératoire de dérivation du liquide céphalorachidien par hôpital pédiatrique ou adulte a montré peu de différence dans les taux d'infection et dans la distribution des pathogènes entre les milieux pédiatriques et adultes/mixtes. Toutefois, une étude antérieure menée entre 2000 et 2002 auprès d'hôpitaux du Programme canadien de surveillance des infections nosocomiales a révélé que les infections du site opératoire de dérivation du liquide céphalorachidien étaient plus fréquentes chez les enfants que chez les adultes (15). Dans cette étude antérieure, le taux d'infection chez les patients pédiatriques était plus élevé que celui observé dans cette étude $(4,9 \%$ des interventions chirurgicales en 2000 à 2002 comparativement à 3,3\% en 2009 à 2018), ce qui indique que les taux d'infections du site opératoire chez les populations pédiatriques ont diminué.

La documentation limitée sur les infections du site opératoire cardiaques pédiatriques, les différences dans les populations de patients et la durée du suivi rendent les comparaisons directes difficiles, mais notre taux global d'infections du site opératoire cardiaques pédiatriques ( $n=4,1$ par 100 chirurgies) est semblable aux fourchettes des taux d'infection déclarées ailleurs. Une étude d'intervention menée en 2009 à 2012 auprès de nouveau-nés subissant une chirurgie cardiaque dans un centre de soins tertiaires à New York a révélé des taux d'infections du site opératoire cardiaque pédiatrique avant et après l'intervention de 6,2 par 100 chirurgies et de 5,8 par 100 chirurgies, respectivement (16). Dans une étude française de 2012 à 2013 portant sur des patients de moins d'un an, $19 \%$ des patients ont présenté une infection du site opératoire (17). Une étude rétrospective menée en 2010 à 2012 sur des patients pédiatriques (de moins de 18 ans) subissant une chirurgie cardiaque dans deux hôpitaux de New York a révélé un taux de 1,4 infection associé aux soins de santé par 100 interventions (18).

Il y a eu une augmentation importante du taux d'infections du site opératoire cardiaque pédiatrique en 2018 , qui est passé à 7,5 par 100 chirurgies. Cette augmentation s'est limitée à deux établissements hospitaliers où des enquêtes sont en cours. II faut interpréter cette augmentation avec prudence, car les taux sont calculés à partir d'un petit nombre de cas et ils peuvent être sensibles aux fluctuations aléatoires dans chaque hôpital. 


\section{Infections du système sanguin associées aux cathéters centraux}

Les infections du système sanguin associées aux cathéters centraux étaient les infections associées aux soins de santé associées aux instruments les plus fréquemment signalées (69\% des infections associées aux soins de santé inclus); toutefois, il est important de noter que le nombre d'hôpitaux participant à la surveillance de chaque infection associée aux soins de santé diffère et que les périodes de surveillance de certaines infections associées aux soins de santé étaient plus courtes. Dans une étude de prévalence ponctuelle des infections associées aux soins de santé, la fréquence des infections du site opératoire (19\%) et des infections du système sanguin associées aux cathéters centraux (21\%) était très semblable (5).

II n'y a pas eu de changements importants dans les taux d'infections du système sanguin associées aux cathéters centraux chez les unités de soins intensifs adultes sondées ou chez unités de soins intensifs pédiatriques; cependant, il y a eu une diminution de $55 \%$ des taux d'infections du système sanguin associées aux cathéters centraux dans les unités de soins intensifs néonatales. Les méthodes de mesure diffèrent, mais les taux d'infections du système sanguin associées aux cathéters centraux dans les unités de soins intensifs néonatales ont également diminué aux États-Unis; entre 2010 et 2016, les ratios d'incidence normalisés (définis comme étant le changement par rapport au nombre d'infections du système sanguin associées aux cathéters centraux par jours (VC) pour les infections du système sanguin associées aux cathéters centraux dans les unités de soins intensifs néonatales et les taux d'utilisation de CVC dans les unités de soins intensifs néonatales ont diminué aux États-Unis (19). De plus, les taux d'infections du système sanguin associées aux cathéters centraux dans d'autres types d'unités de soins intensifs aux États-Unis ont également diminué entre 2010 et 2016 (19). La réduction des taux aux États-Unis a été attribuée à la mise à jour des lignes directrices du National Healthcare Surveillance Network (20). Il est possible que des améliorations aient été apportées aux taux au Canada avant la période à l'étude.

Nos taux globaux d'infections du système sanguin associées aux cathéters centraux dans les unités de soins intensifs pour adultes (0,7 et 1,1 par 1000 jours CVC pour les unités de soins intensifs en chirurgie cardiovasculaire et les unités de soins intensifs mixtes, respectivement) sont semblables aux fourchettes déclarées aux États-Unis et en Australie. Aux États-Unis, le taux d'infections du système sanguin associées aux cathéters centraux dans les unités de soins intensifs a été estimé à 0,8 par 1000 jours CVC en 2010 à 2015 (21). En Australie, les taux annuels d'infections du système sanguin associées aux cathéters centraux dans les unités de soins intensifs se situaient entre 0,9 et 1,7 par 1000 jours CVC en 2010 à 2013 (22). Les taux sont plus élevés dans d'autres régions; une vaste étude de surveillance portant sur 703 unités de soins intensifs en Amérique latine, en Europe, en Méditerranée orientale, en Asie du Sud-Est et dans l'ouest du Pacifique a révélé un taux d'infections du système sanguin associées aux cathéters centraux de 4,1 par 1000 jours CVC entre janvier 2010 et décembre 2015 (21).

\section{Antibiogramme}

Le pourcentage d'isolats de $S$. aureus résistant à la méthicilline dans cette étude (13\%) est semblable à ce qui a été signalé dans un réseau de surveillance suisse où $8 \%$ des cas de $S$. aureus résistant à la méthicilline de $S$. aureus étaient des cas de S. aureus résistant à la méthicilline en 2010 à 2015 (23). Des taux plus élevés de $S$. aureus résistant à la méthicilline ont été signalés ailleurs. Aux États-Unis, de $42 \%$ à $48 \%$ des isolats de $S$. aureus des infections associées aux soins de santé (notamment les infections du site opératoire, les infections du système sanguin associées aux cathéters centraux et autres) dans le cadre de la surveillance du National Healthcare Surveillance Network étaient des $S$. aureus résistant à la méthicilline (5). Une étude japonaise sur les infections du site opératoire dans 27 centres médicaux a révélé que $72 \%$ des isolats de $S$. aureus étaient des $S$. aureus résistant à la méthicilline en 2010 (24).

Parmi les Enterococcus spp. décelés, $17 \%$ étaient des entérocoques résistants à la vancomycine dans le cadre de notre surveillance. Dans le cadre de la surveillance du National Healthcare Surveillance Network aux États-Unis, 8,5\% des agents pathogènes Enterococcus faecalis et $84,5 \%$ des agents pathogènes Enterococcus fæcium identifiés dans les infections du système sanguin associées aux cathéters centraux des unités de soins intensifs étaient des entérocoques résistants à la vancomycine en 2015 à 2017 (5).

La résistance au méropénème était faible chez les pathogènes à Gram négatif, avec deux isolats de Klebsiella sur 36 (6\%) et un isolat d'E. coli sur $33(3 \%)$ résistants au méropénème. Aux États-Unis, le pourcentage d'entérobactéries résistantes au carbapénème parmi les Klebsiella spp. variait de 3,1\% (parmi les infections du site opératoire) à 6,9\% (parmi la liste élargie des infections associées aux instruments); le pourcentage d'entérobactéries résistantes au carbapénème parmi les $E$. coli variait de $0,6 \%$ (parmi les infections du site opératoire) à $0,7 \%$ (parmi la liste élargie des infections associées aux instruments) (5).

\section{Force et faiblesse}

La force de cette étude réside dans la collecte normalisée de données détaillées provenant d'un vaste réseau d'hôpitaux sentinelles pendant une décennie. Bien que le réseau du Programme canadien de surveillance des infections nosocomiales s'étende à l'ensemble du Canada, les hôpitaux participants ne sont peut-être pas représentatifs de la population générale des patients hospitalisés au Canada; les hôpitaux participant au Programme canadien de surveillance des infections nosocomiales ont tendance à être des hôpitaux plus grands qui donnent de la formation dans des centres urbains. Le Programme canadien 
de surveillance des infections nosocomiales fait actuellement l'objet d'un processus de recrutement visant à accroître la représentativité et la couverture des lits, en particulier dans les populations nordiques, rurales et autochtones. Les données du Programme canadien de surveillance des infections nosocomiales, bien qu'elles soient normalisées, peuvent être sensibles aux changements dans la participation des hôpitaux, les pratiques de prévention et de contrôle des infections et l'application des définitions de surveillance. Les différences entre les protocoles de surveillance et les définitions de cas limitent la capacité de comparer les données d'autres pays. Toutefois, les données présentées dans le présent rapport sont couramment utilisées par les hôpitaux canadiens à des fins d'analyse comparative.

En ce qui concerne la surveillance des infections du système sanguin associées aux cathéters centraux, nous n'avons pas de données sur les infections survenant à l'extérieur des unités de soins intensifs; toutefois, aux États-Unis, les infections du système sanguin associées aux cathéters centraux à l'extérieur du contexte des unités de soins intensifs représentaient $55 \%$ de toutes les infections du système sanguin associées aux cathéters centraux (19).

\section{Conclusion}

Ce rapport fournit un résumé à jour des taux, de la distribution des agents pathogènes et de la résistance aux antimicrobiens parmi les infections associées aux soins de santé associées à certains instruments et les agents pathogènes pertinents. II est essentiel de comprendre le fardeau national des infections associées aux soins de santé associées aux instruments pour élaborer et maintenir des taux de référence afin d'éclairer les politiques et les programmes de contrôle des infections et de prévention des antimicrobiens.

\section{Déclaration des auteurs}

Les hôpitaux du Programme canadien de surveillance des infections nosocomiales ont fourni une expertise dans l'élaboration de protocoles en plus de la présentation de données épidémiologiques. Les épidémiologistes de l'Agence de la santé publique du Canada étaient responsables de la conception, de l'analyse, de l'interprétation, de la rédaction et de la révision de ce document.

\section{Conflits d'intérêts}

Aucun.

\section{Remerciements}

Nous sommes reconnaissants de la contribution des médecins, des épidémiologistes, des praticiens du contrôle des infections et du personnel de laboratoire de chaque hôpital participant :

Vancouver General Hospital, Vancouver, (Colombie-Britannique [C.-B.]); Richmond General Hospital, Richmond (C.-B.); UBC Hospital, Vancouver (C.-B.); Lions Gate, North Vancouver (C.-B.); Powell River General Hospital, Powell River (C.-B.); Sechelt Hospital (anciennement St. Mary's), Sechelt (C.-B.); Squamish General Hospital, Squamish (C.-B.); Peter Lougheed Centre, Calgary (Alberta [Alb.]); Rockyview General Hospital, Calgary (Alb.); South Health Campus Calgary (Alb.); Foothills Medical Centre, Calgary (Alb.); Alberta Children's Hospital, Calgary (Alb.); University of Alberta Hospital, Edmonton (Alb.); Stollery Children's Hospital, Edmonton (Alb.); Health Sciences Centre-Winnipeg, Winnipeg (Manitoba [Man.]); University of Manitoba Children's Hospital, Winnipeg (Man.); Children's Hospital of Western Ontario, London (Ontario [Ont.]); Victoria Hospital, London (Ont.); University Hospital, London (Ont.); Toronto General Hospital, Toronto (Ont.); Toronto Western Hospital, Toronto (Ont.); Princess Margaret Hospital, Toronto (Ont.); Mount Sinai Hospital, Toronto (Ont.); Bridgepoint Active Healthcare, Toronto (Ont.); Sunnybrook Hospital, Toronto (Ont.); Kingston General Hospital, Kingston (Ont.); Hôpital général juif - Sir Mortimer B. Davis, Montréal (Québec [Qc.]); Moncton Hospital, Moncton (Nouveau-Brunswick); Halifax Infirmary, Halifax (Nouvelle-Écosse [N.-É.]); Victoria General, Halifax (N.-É.); Centre de réadaptation, Halifax (N.-É.); Édifice commémoratif des anciens combattants, Halifax (N.-É.); Dartmouth General Hospital, Halifax (N.-É.); IWK Health Centre, Halifax (N.-É.); Hospital for Sick Children, Toronto (Ont.); Hôpital de Montréal pour enfants, Montréal (Qc.); Royal University Hospital, Saskatoon (Saskatchewan [Sask.]); Hôpital St. Paul, Saskatoon (Sask.); General Hospital \& Miller Centre, St. John's (Terre-Neuve-et-Labrador [T.-N.-L.]); Burin Peninsula Health Care Centre, Burin (T.-N.-L.); Carbonear General Hospital, Carbonear (T.-N.-L.); Dr. G.B. Cross Memorial Hospital, Cross Memorial Hospital, Clarenville (T.-N.-L.); Janeway Children's Hospital and Rehabilitation Centre, St. John's (T.-N.-L.); St Clare's Mercy Hospital, St. John's (T.-N.-L.); McMaster Children's Hospital, Hamilton (Ont.); St. Joseph's Healthcare, Hamilton (Ont.); Jurvinski Hospital and Cancer Center, Hamilton (Ont.); General Site, Hamilton (Ont.); Civic Campus, Ottawa (Ont.); General Campus, Ottawa (Ont.); University of Ottawa Heart Institute, Ottawa (Ont.); Hôpital Maisonneuve-Rosemont, Montréal (Qc.); Victoria General Hospital, Victoria (C.-B.); Royal Jubilee, Victoria (C.-B.); Nanaimo Regional General Hospital, Nanaimo (C.-B.); Children's Hospital of Eastern Ontario, Ottawa (Ont.); BC Women's Hospital, Vancouver (C.-B.); Hôtel-Dieu de Québec, Québec (Qc.); Hôpital général de Montréal, Montréal (Qc.); Hôpital Royal Victoria, Montréal (Qc.); Institut et hôpital neurologiques de Montréal, Montréal (Qc.); North York General Hospital, Toronto (Ont.); Kelowna General Hospital, Kelowna (C.-B.); Queen Elizabeth Hospital, Charlottetown 
(île-du-Prince-Édouard [î.-P.-É.]); Prince County Hospital, Summerside (Î.-P.-É.); Western Memorial Regional Hospital, Corner Brook (T.-N.-L.); Regina General Hospital, Regina (Sask.); Pasqua Hospital, Regina (Sask.); Sudbury Regional Hospital, Sudbury (Ont.); University of Northern BC, Prince George (C.-B.).

Merci au personnel de l'Agence de la santé publique du Canada au Centre de contrôle des maladies transmissibles et des infections, Ottawa (Ontario) (J. Brooks, L. Pelude, R. Mitchell, W. Rudnick, K. B. Choi, A. Silva, V. Steele, J. Cayen, C. McClellan, J. Liang, M. Hunt et L. Sauvé) et au Laboratoire national de microbiologie, Winnipeg (Manitoba) (G. Golding, M. Mulvey, J. Campbell, T. Du, M. McCracken, L. Mataseje, A. Bharat et D. Boyd).

\section{Financement}

Ce travail a été appuyé par l'Agence de la santé publique du Canada.

\section{Références}

1. Allegranzi B, Nejad SB, Castillejos GG, Kilpatrick C, Kelley EM. WHO Guidelines on Hand Hygiene in Health Care: First Global Patient Safety Challenge Celan Care is Safer Care. Chapter 3. The burden of health care-associated infection. Geneva (CH): WHO; 2011. https://www.ncbi.nlm.nih.gov/ books/NBK144030/

2. Al-Tawfiq JA, Tambyah PA. Healthcare associated infections (HAl) perspectives. J Infect Public Health 2014;7(4):339-44. DOI PubMed

3. Institut canadien pour la sécurité des patients. Vérification nationale sur la prévention des infections du site opératoire. 2016 février. ICSP; 2016. https://www.patientsafetyinstitute. $\mathrm{ca} / \mathrm{fr} /$ toolsResources/Documents/SSI\%20Audit\%202016_ Recap\%20Report\%20FR.pdf

4. Mitchell R, Taylor G, Rudnick W, Alexandre S, Bush K, Forrester L, Frenette C, Granfield B, Gravel-Tropper D, Happe J, John M, Lavallee C, McGeer A, Mertz D, Pelude L, Science M, Simor A, Smith S, Suh KN, Vayalumkal J, Wong A, Amaratunga K; Canadian Nosocomial Infection Surveillance Program. Trends in health care-associated infections in acute care hospitals in Canada: an analysis of repeated point-prevalence surveys. CMAJ 2019;191(36):E981-8. DOI PubMed

5. Weiner-Lastinger LM, Abner S, Edwards JR, Kallen AJ, Karlsson M, Magill SS, Pollock D, See I, Soe MM, Walters MS, Dudeck MA. Antimicrobial-resistant pathogens associated with adult healthcare-associated infections: Summary of data reported to the National Healthcare Safety Network, 2015-2017. Infect Control Hosp Epidemiol 2020;41(1):1-18. DOl PubMed
6. Zimlichman E, Henderson D, Tamir O, Franz C, Song $P$, Yamin CK, Keohane C, Denham CR, Bates DW. Health care-associated infections: a meta-analysis of costs and financial impact on the US health care system. JAMA Intern Med 2013;173(22):2039-46. DOI PubMed

7. Nodzo SR, Frisch NB. The Use of Antibiograms in Orthopedic Surgery. Curr Rev Musculoskelet Med 2019;11(3):341-6. DOI PubMed

8. Center for Disease and Prevention Control. National Healthcare Safety Network (NHSN) Overview Patient Safety Component Manual. 2020. https://www.cdc.gov/nhsn/pdfs/ pscmanual/pcsmanual_current.pdf

9. European Centre for Disease Prevention and Control. Healthcare-associated infections: surgical site infections Annual Epidemiological Report for 2017. Solna

(Sweden): ECDC; 2019. https://www.ecdc.europa.eu/en/ publications-data/healthcare-associated-infections-surgica |-site-infections-annual-1

10. Magill SS, O'Leary E, Janelle SJ, Thompson DL, Dumyati G, Nadle J, Wilson LE, Kainer MA, Lynfield R, Greissman S, Ray SM, Beldavs Z, Gross C, Bamberg W, Sievers M, Concannon C, Buhr N, Warnke L, Maloney M, Ocampo V, Brooks J, Oyewumi T, Sharmin S, Richards K, Rainbow J, Samper M, Hancock EB, Leaptrot D, Scalise E, Badrun F, Phelps R, Edwards JR; Emerging Infections Program Hospital Prevalence Survey Team. Changes in prevalence of health care-associated infections in U.S. Hospitals. N Engl J Med 2018;379(18):1732-44. DOI PubMed

11. Public Health England. Surveillance of surgical site infections in NHS hospitals in England, 2017 to 2018. London (UK): PHE; 2018. https://assets.publishing.service.gov.uk/ government/uploads/system/uploads/attachment_data/ file/765967/SSI_annual_report_NHS_hospitals_2017_18.pdf

12. Kandel CE, Jenkinson R, Daneman N, Backstein D, Hansen BE, Muller MP, Katz KC, Widdifield J, Bogoch E, Ward S, Sajja A, Jeldes FG, McGeer A. Predictors of Treatment Failure for Hip and Knee Prosthetic Joint Infections in the Setting of 1- and 2-Stage Exchange Arthroplasty: A Multicenter Retrospective Cohort. Open Forum Infect Dis 2019;6(11):ofz452. DOl PubMed

13. Springer BD, Cahue S, Etkin CD, Lewallen DG, McGrory BJ. Infection burden in total hip and knee arthroplasties: an international registry-based perspective. Arthroplast Today 2017;3(2):137-40. DOl PubMed

14. Wong JM, Ziewacz JE, Ho AL, Panchmatia JR, Bader AM, Garton HJ, Laws ER, Gawande AA. Patterns in neurosurgical adverse events: cerebrospinal fluid shunt surgery. Neurosurg Focus 2012;33(5):E13. DOI PubMed

15. Langley JM, Gravel D, Moore D, Matlow A, Embree J, MacKinnon-Cameron D, Conly J; Canadian Nosocomial Infection Surveillance Program. Study of cerebrospinal fluid shunt-associated infections in the first year following placement, by the Canadian Nosocomial Infection Surveillance Program. Infect Control Hosp Epidemiol 2009;30(3):285-8. DOI PubMed 
16. Murray MT, Corda R, Turcotte R, Bacha E, Saiman L, Krishnamurthy G. Implementing a standardized perioperative antibiotic prophylaxis protocol for neonates undergoing cardiac surgery. Ann Thorac Surg 2014;98(3):927-33. DOl PubMed

17. Macher J, Gras Le Guen C, Chenouard A, Liet JM, Gaillard Le Roux B, Legrand A, Mahuet J, Launay E, Gournay V, Joram N. Preoperative Staphylococcus aureus Carriage and Risk of Surgical Site Infection After Cardiac Surgery in Children Younger than 1 year: A Pilot Cohort Study. Pediatr Cardiol 2017;38(1):176-83. DOI PubMed

18. Turcotte RF, Brozovich A, Corda R, Demmer RT, Biagas $K V$, Mangino $D$, Covington $L$, Ferris $A$, Thumm $B$, Bacha E, Smerling A, Saiman L. Health care-associated infections in children after cardiac surgery. Pediatr Cardiol 2014;35(8):1448-55. DOI PubMed

19. Centers for Disease Control and Prevention. Data Summary of HAls in the US: Assessing Progress 20062016. CDC; 2017. https://www.cdc.gov/hai/data/archive/ data-summary-assessing-progress.html

20. Bell T, O'Grady NP. Prevention of Central Line-Associated Bloodstream Infections. Infect Dis Clin North Am 2017;31(3):551-9. DOl PubMed

21. Rosenthal VD, Al-Abdely HM, El-Kholy AA, AIKhawaja SA, Leblebicioglu H, Mehta Y, Rai V, Hung NV, Kanj SS, Salama MF, Salgado-Yepez E, Elahi N, Morfin Otero R, Apisarnthanarak A, De Carvalho BM, Ider BE, Fisher D, Buenaflor MC, Petrov MM, Quesada-Mora AM, Zand F, Gurskis V, Anguseva T, Ikram A, Aguilar de Moros D, Duszynska W, Mejia N, Horhat FG, Belskiy V, Mioljevic V, Di Silvestre G, Furova K, Ramos-Ortiz GY, Gamar Elanbya MO, Satari HI, Gupta U, Dendane T, Raka L, Guanche-Garcell H, Hu B, Padgett D, Jayatilleke K, Ben Jaballah N, Apostolopoulou E, Prudencio Leon WE, Sepulveda-Chavez A, Telechea HM, Trotter A, Alvarez-Moreno C, Kushner-Davalos L; Remaining authors. International Nosocomial Infection Control Consortium report, data summary of 50 countries for 2010-2015: device-associated module. Am J Infect Control 2016;44(12):1495-504. DOI PubMed
22. Spelman T, Pilcher DV, Cheng AC, Bull AL, Richards MJ, Worth LJ. Central line-associated bloodstream infections in Australian ICUs: evaluating modifiable and non-modifiable risks in Victorian healthcare facilities. Epidemiol Infect 2017;145(14):3047-55. DOI PubMed

23. Abbas M, Aghayev E, Troillet N, Eisenring MC, Kuster SP, Widmer AF, Harbarth S; SwissNoso. Temporal trends and epidemiology of Staphylococcus aureus surgical site infection in the Swiss surveillance network: a cohort study. J Hosp Infect 2018;98(2):118-26. DOI PubMed

24. Takesue $Y$, Watanabe A, Hanaki H, Kusachi S, Matsumoto T, Iwamoto A, Totsuka K, Sunakawa K, Yagisawa M, Sato J, Oguri T, Nakanishi K, Sumiyama Y, Kitagawa Y, Wakabayashi G, Koyama I, Yanaga K, Konishi T, Fukushima R, Seki S, Imai S, Shintani T, Tsukada H, Tsukada K, Omura K, Mikamo H, Takeyama H, Kusunoki M, Kubo S, Shimizu J, Hirai T, Ohge H, Kadowaki A, Okamoto K, Yanagihara K. Nationwide surveillance of antimicrobial susceptibility patterns of pathogens isolated from surgical site infections (SSI) in Japan. J Infect Chemother 2012 Dec;18(6):816-26. DOI PubMed 


\section{Annexe 1 : Définitions de cas}

\section{Infections du système sanguin associées aux cathéters centraux}

Seules les infections du système sanguin associées aux cathéters centraux liées à une admission à une unité de soins intensifs ont été incluses dans le cadre de la surveillance.

\section{Définition de cas d'infections du système sanguin :}

Les infections du système sanguin ne sont PAS liées à une infection à un autre site et répondent à l'un des critères suivants :

Critère 1 : Agent pathogène reconnu, cultivé à partir d'au moins une hémoculture, sans lien avec une infection sur un autre site.

\section{OU}

Critère 2 : Au moins un des symptômes suivants : fièvre (plus de $38{ }^{\circ} \mathrm{C}$ température centrale), frissons, hypotension (si patient âgé de plus de 1 an : fièvre [plus de $38^{\circ} \mathrm{C}$ température centrale], hypothermie [moins de $36^{\circ} \mathrm{C}$ température centrale], apnée ou bradycardie) ET contaminant cutané courant (voir la liste ci-dessous) cultivé à partir d'au moins deux échantillons de sang prélevés lors de deux occasions distinctes ou à des sites différents sans rapport avec une infection à un autre site. Les différents sites peuvent comprendre des veines périphériques, des cathéters de voie centrale ou les lumières séparées d'un cathéter multi lumière. Les différents moments comprennent deux hémocultures prélevées le même jour ou des jours civils consécutifs par des ponctions veineuses ou des entrées de cathéter séparées. La date de prélèvement de la première culture sanguine positive est la date utilisée pour identifier la date de la culture positive. Deux flacons d'hémoculture positive remplis à la même entrée de ponction veineuse ou de cathéter ne constituent qu'une seule hémoculture positive.

Définition de cas d'infection du système sanguin associée aux cathéters centraux :

Une infection du système sanguin confirmée en laboratoire pour laquelle un cathéter de voie centrale ou un cathéter ombilical était en place depuis plus de deux jours civils à la date de I'hémoculture positive, le jour du placement du dispositif étant le premier jour. En cas d'admission ou de transfert dans un établissement où un cathéter de voie centrale cathéter ombilical est en place (e.g. un cathéter central tunnelisé ou implanté), le jour du premier accès est considéré comme le jour 1 .

\section{ET}

Une infection où le cathéter de voie centrale ou cathéter ombilical était en place depuis plus de deux jours civils, puis retiré le jour ou un jour avant le prélèvement d'une culture sanguine positive. Si un cathéter de voie centrale ou un cathéter ombilical a été en place pendant plus de deux jours civils et qu'il a été retiré, les critères de l'infection du système sanguin doivent être entièrement respectés le jour du retrait ou le jour suivant. Si le patient est admis ou transféré dans l'unité de soins intensifs avec un cathéter de voie centrale en place, le jour du premier accès est considéré comme le jour 1. L'accès est défini comme la mise en place, la perfusion ou le retrait de la voie.

Définition des cas liés aux soins intensifs :

Apparition de l'infection du système sanguin associée aux cathéters centraux pendant le séjour aux soins intensifs et le cathéter de voie centrale est en place depuis plus de deux jours civils. L'infection du système sanguin associée aux cathéters centraux serait attribuable à l'unité de soins intensifs si elle s'est produite le jour du transfert ou le jour civil suivant le transfert à l'extérieur de l'unité de soins intensifs.

\section{Contaminants cutanés courants :}

Diphtéroïdes, Corynebacterium spp., Bacillus spp., Propionibacterium spp., staphylocoques négatifs à la coagulase (y compris S. epidermidis), streptocoques du groupe des viridans, Aerococcus spp., Micrococcus spp. et Rhodococcus spp.

\section{Infection du site opératoire de la hanche et du genou}

Seules les infections complexes du site opératoire (infections du site opératoire profonde associée à une incision ou organe/ espace) suivant une arthroplastie de la hanche et du genou ont été incluses dans la surveillance.

Une infection du site opératoire profonde associée à une incision doit répondre au critère suivant :

L'infection survient dans les 90 jours suivant l'intervention chirurgicale et l'infection semble être liée à l'intervention chirurgicale et touche les tissus mous profonds (e.g. couches faciales et musculaires) de l'incision, et le patient a au moins UN des symptômes suivants :

1. Drainage purulent de l'incision profonde, mais pas de l'organe/espace composant le site chirurgical.

2. L'incision profonde est spontanément déhiscente ou est délibérément ouverte par le chirurgien et est culturepositive ou non-cultivée et le patient présente au moins un des signes ou symptômes suivants : fièvre (plus de $38^{\circ} \mathrm{C}$ ), ou douleur ou sensibilité localisée. Un résultat de culture négative ne répond pas à ce critère.

3. Un abcès ou autre preuve d'infection impliquant l'incision profonde est trouvé lors d'un examen direct, lors d'une réopération ou par un examen histopathologique ou radiologique. 
4. Diagnostic d'une incision profonde par un chirurgien ou un médecin traitant.

Une infection du site opératoire d'organe/espace doit répondre au critère suivant :

L'infection survient dans les 90 jours suivant la procédure opératoire et semble être liée à la procédure opératoire et l'infection concerne toute partie du corps, à l'exception de I'incision cutanée, du fascia ou des couches musculaires, qui est ouverte ou manipulée pendant la procédure opératoire et dont le patient présente au moins UN des éléments suivants :

1. Drainage purulent d'un drain placé dans l'organe/espace à travers une plaie.

2. Organismes isolés à partir d'une culture de fluide ou de tissu dans l'organe/espace à des fins de diagnostic ou de traitement clinique.

3. Un abcès ou autre preuve d'infection impliquant l'organe/espace qui est trouvé lors d'un examen direct, pendant une réopération ou par un examen histopathologique ou radiologique.

4. Diagnostic d'une infection du site opératoire de l'organe/ espace par un chirurgien ou un médecin traitant.

\section{Infection du site opératoire des voies de dérivation du liquide céphalorachidien}

Seuls les patients qui ont subi une mise en place ou une révision d'un instrument d'aiguillage de liquide céphalorachidien et dont l'infection s'est produite dans l'année suivant l'intervention chirurgicale ont été inclus dans la surveillance.

\section{Définition de cas d'infection du site opératoire des voies de} dérivation du liquide céphalorachidien :

Un patient est signalé comme ayant une infection du site opératoire de dérivation du liquide céphalorachidien s'il répond aux critères suivants :

Critère 1 : Un dispositif interne de dérivation du liquide céphalorachidien est en place

\section{ET}

Critère 2 : Un agent pathogène bactérien ou fongique est décelé dans le liquide céphalorachidien

\section{ET}

Critère 3 : L'agent pathogène est associé à au moins UNE des manifestations suivantes :

1. Fièvre (température $d^{\prime}$ au moins $38^{\circ} \mathrm{C}$ )

2. Signes ou symptômes neurologiques

3. Signes ou symptômes abdominaux

4. Signes ou symptômes d'une défaillance ou d'une obstruction du dispositif de dérivation

\section{Infection du site opératoire après une opération cardiaque pédiatrique}

Seules les infections du site opératoire après une opération à cœur ouvert avec pontage cardiopulmonaire chez les patients pédiatriques (plus de 18 ans) ont été incluses dans le cadre de la surveillance.

Une infection du site opératoire profonde associée à une incision doit répondre au critère suivant :

L'infection se produit dans les 30 jours suivant l'intervention chirurgicale et ne concerne que la peau et le tissu sous-cutané de I'incision et répond à au moins UN des critères suivants :

1. Drainage purulent de l'incision superficielle.

2. Organismes isolés à partir d'une culture de liquide ou de tissu provenant de l'incision superficielle.

3. Le patient présente au moins UN des signes ou symptômes d'infection suivants :

- Douleur ou sensibilité localisée; gonflement localisé; érythème; ou chaleur et incision superficielle délibérément ouverte par un chirurgien, et l'incision superficielle ou les tissus sous-cutanés sont culturepositive ou non cultivée. Un résultat de culture négative ne répond pas à ce critère

- Diagnostic d'une lésion superficielle par incision par le chirurgien ou le médecin traitant

Une infection du site opératoire profonde associée à une incision doit répondre au critère suivant :

L'infection se produit dans les 90 jours suivant l'intervention chirurgicale et l'infection semble être liée à l'intervention chirurgicale ET concerne les tissus mous profonds (e.g. les couches du visage et des muscles) de l'incision ET le patient présente au moins UN des éléments suivants :

1. Drainage purulent de l'incision profonde, mais pas de l'organe/espace composant le site chirurgical.

2. L'incision profonde est spontanément déhiscente ou est délibérément ouverte par le chirurgien, et est culturepositive ou non-cultivée et le patient présente au moins un des signes ou symptômes suivants : fièvre (plus de $38^{\circ} \mathrm{C}$ ), douleur ou sensibilité localisée. Un résultat de culture négative ne répond pas à ce critère.

3. Un abcès ou autre preuve d'infection impliquant l'incision profonde est trouvé lors d'un examen direct, lors d'une réopération ou par un examen histopathologique ou radiologique.

4. Diagnostic d'une incision profonde par un chirurgien ou un médecin traitant. 
Une infection du site opératoire d'organe/espace doit répondre au critère suivant :

1. L'infection se produit dans les 90 jours après l'intervention chirurgicale et l'infection semble être liée à l'intervention chirurgicale ET l'infection concerne toute partie du corps plus profonde que les couches fasciales/musculaires qui est ouverte ou manipulée pendant l'intervention chirurgicale ET le patient présente au moins UN des éléments suivants :

2. Drainage purulent d'un drain placé dans l'organe/espace à travers une plaie.
3. Organismes isolés à partir d'une culture de fluide ou de tissu dans l'organe/espace à des fins de diagnostic ou de traitement clinique.

4. Un abcès ou autre preuve d'infection impliquant l'organe/espace qui est trouvé lors d'un examen direct, pendant une réopération ou par un examen histopathologique ou radiologique.

5. Diagnostic d'une infection du site opératoire de l'organe/espace par un chirurgien ou un médecin traitant. 